\title{
On Six-Parameter Fréchet Distribution: Properties and Applications
}

\author{
Haitham M. Yousof \\ Department of Statistics, Mathematics and Insurance \\ Benha University, Egypt \\ haitham.yousof@fcom.bu.edu.eg
}

\author{
Ahmed Z. Afify \\ Department of Statistics, Mathematics and Insurance \\ Benha University, Egypt \\ Ahmed.afify@fcom.bu.edu.eg \\ Abd El Hadi N. Ebraheim \\ Institute of Statistical Studies \& Research \\ Cairo University, Egypt \\ dr.hadi@cu.edu.eg \\ G. G. Hamedani \\ Department of Mathematics, Statistics and Computer Science \\ Marquette University, USA \\ gholamhoss.hamedani@marquette.edu \\ Nadeem Shafique Butt \\ Department of Family and Community Medicine, \\ King Abdulaziz University, Jeddah, Kingdom of Saudi Arabia \\ nshahfique@kau.edu.sa
}

\begin{abstract}
This paper introduces a new generalization of the transmuted Marshall-Olkin Fréchet distribution of Afify et al. (2015), using Kumaraswamy generalized family. The new model is referred to as Kumaraswamy transmuted Marshall-Olkin Fréchet distribution. This model contains sixty two sub-models as special cases such as the Kumaraswamy transmuted Fréchet, Kumaraswamy transmuted Marshall-Olkin, generalized inverse Weibull and Kumaraswamy Gumbel type II distributions, among others. Various mathematical properties of the proposed distribution including closed forms for ordinary and incomplete moments, quantile and generating functions and Rényi and $\eta$-entropies are derived. The unknown parameters of the new distribution are estimated using the maximum likelihood estimation. We illustrate the importance of the new model by means of two applications to real data sets.
\end{abstract}

Keywords: Moments of residual life, Goodness-of-fit, Order Statistics, Maximum Likelihood Estimation.

\section{Introduction}

The procedure of expanding a family of distributions for added flexibility or to construct covariate models is a well-known technique in the literature. In many applied sciences such as medicine, engineering and finance, amongst others, modeling and analyzing lifetime data are crucial. Several lifetime distributions have been used to model such kinds of data. The quality of the procedures used in a statistical analysis depends heavily on the assumed probability model or distributions. Because of this, considerable effort 
has been expended in the development of large classes of standard probability distributions along with relevant statistical methodologies. However, there still remain many important problems where the real data does not follow any of the classical or standard probability models. The Fréchet distribution is one of the important distributions in extreme value theory and it has been applied to data on characteristics of sea waves and wind speeds. Further information about the Fréchet distribution and its applications were discussed in Kotz and Nadarajah (2000).

Recently, some extensions of the Fréchet distribution are considered. The exponentiated Fréchet (Nadarajah and Kotz, 2003), beta Fréchet (Nadarajah and Gupta, 2004 and Barreto-Souza et al., 2011), transmuted Fréchet (Mahmoud and Mandouh, 2013), Marshall-Olkin Fréchet (Krishna et.al., 2013), gamma extended Fréchet (da Silva et al., 2013), transmuted exponentiated Fréchet (Elbatal et al. 2014), Kumaraswamy Fréchet (Mead and Abd-Eltawab, 2014), transmuted Marshall-Olkin Fréchet (TMOF) (Afify et al., 2015), transmuted exponentiated generalized Fréchet (Yousof et al., 2015), beta exponential Fréchet (Mead et al., 2016) and Weibull Fréchet (Afify et al., 2016) distributions. The cumulative distribution function (cdf) of the TMOF distribution is given (for $x>0$ ) by

$$
F(x)=\frac{\alpha(1+\lambda) \exp \left[-\left(\frac{\theta}{x}\right)^{\beta}\right]-(\alpha \lambda+\alpha-1) \exp \left[-2\left(\frac{\theta}{x}\right)^{\beta}\right]}{\left\{\alpha+(1-\alpha) \exp \left[-\left(\frac{\theta}{x}\right)^{\beta}\right]\right\}^{2}}
$$

where $\alpha, \beta$ and $\theta$ are positive, $|\lambda| \leq 1, \theta$ is a scale parameter, $\alpha, \beta$ and $\lambda$ are shape parameters. The corresponding probability density function (pdf)is given by

$$
f(x)=\frac{\frac{\alpha \beta}{\theta}\left(\frac{\theta}{x}\right)^{\beta+1} \exp \left[-\left(\frac{\theta}{x}\right)^{\beta}\right]\left\{\alpha(1+\lambda)-(\alpha \lambda+\alpha+\lambda-1) \exp \left[-\left(\frac{\theta}{x}\right)^{\beta}\right]\right\}}{\left\{\alpha+(1-\alpha) \exp \left[-\left(\frac{\theta}{x}\right)^{\beta}\right]\right\}^{3}} .
$$

In this article we present a new generalization of the TMOF distribution called Kumaraswamy transmuted Marshall-Olkin Fréchet (Kw-TMOF) distribution based on the family of Kumaraswamy generalized (Kw-G) distributions introduced by Cordeiro and de Castro (2011). The main motivation for this extension is that the new distribution is a highly flexible life distribution which contains as sub models sixty two well known and unknown distributions, admits different degrees of kurtosis and asymmetry and the Kumaraswamy transmuted Marshall-Olkin Fréchet (Kw-TMOF) distribution provides a superior fit to real data than its sub models and non-nested models.

Definition 1. A random variable $\mathrm{X}$ is said to have $\mathrm{Kw}-\mathrm{G}$ distribution if its cdf is given by

$$
F(x)=1-\left[1-G(x)^{a}\right]^{b},
$$

where $a>0$ and $b>0$ are two additional parameters whose role is to introduce skewness and to vary tail weights. The corresponding pdf is given by

$$
f(x)=\operatorname{abg}(x) G(x)^{a-1}\left[1-G(x)^{a}\right]^{b-1},
$$

where $G(x)$ and $g(x)$ are the cdf and pdf of the baseline distribution respectively. Clearly when $a=b=1$, we obtain the baseline distribution. 
Providing a new class of distributions is always precious for statisticians. Thus, the aim of this paper is to study the $\mathrm{Kw}-\mathrm{TMOF}$ distribution. The fact that the $\mathrm{Kw}-\mathrm{TMOF}$ distribution generalizes existing commonly used distributions and introduces new lifetime models is an important aspect of the model. Further, we demonstrate that the proposed model provides a significant improvement compared to some existing lifetime models and it is also a competitive model to the gamma extended Fréchet (da Silva et al., 2013) and beta Fréchet (Barreto-Souza et al., 2011) distributions. In addition, we investigate some mathematical properties of the new model, discuss maximum likelihood estimation of its parameters and derive the observed information matrix.

The rest of the paper is outlined as follows. In Section 2, we demonstrate the subject distribution and the expantions for the pdf and cdf. The statistical properties include quantile functions, random number generation, moments, moment generating functions, incomplete moments, mean deviations and Rényi and $\eta$-entropies are derived in Section 3. The order statistics and their moments are investigated in Section 4. The characterization of the Kw-TMOF in terms of a truncated moment of a function of the random variable is given in Section 5. In Section 6, We discuss maximum likelihood estimation of the model parameters. In Section 7, the Kw-TMOF distribution is applied to two real data sets to illustrate the potentiality of the new distribution for lifetime data modeling. Finally, we provide some concluding remarks in Section 8.

\section{The Kw-TMOF Distribution}

The Kw-TMOF distribution and its sub-models are presented in this section. A random variable $X$ is said to have Kw-TMOF with vector parameters $\phi$, where $\phi=(\alpha, \beta, \theta, \lambda, a, b)$, if its cdf is defined (for $x>0)$ by

$F(x, \phi)=$

$$
1-\left\{-\left[\alpha(1+\lambda) e^{-\left(\frac{\theta}{x}\right)^{\beta}}-(\alpha \lambda+\alpha-1) e^{-2\left(\frac{\theta}{x}\right)^{\beta}}\right]^{a}\left[\alpha+(1-\alpha) e^{-\left(\frac{\theta}{x}\right)^{\beta}}\right]^{-2 \mathrm{a}}\right\}^{b}
$$

where $a, b$ are two additional shape parameters. The corresponding pdf of the Kw-TMOF is given by

$$
\begin{aligned}
& f(x, \phi)=a b \alpha \beta \theta^{\beta} x^{-\beta-1} \frac{\alpha(1+\lambda) e^{-\left(\frac{\theta}{x}\right)^{\beta}}-(\alpha \lambda+\alpha+\lambda-1) e^{-2\left(\frac{\theta}{x}\right)^{\beta}}}{\left[\alpha+(1-\alpha) e^{\left.-\left(\frac{\theta}{x}\right)^{\beta}\right]^{2 a+1}}\right.} \\
& \quad \times\left[\frac{\alpha(1+\lambda)}{e^{2\left(\frac{\theta}{x}\right)^{\beta}}}-\frac{(\alpha \lambda+\alpha-1)}{\alpha}\right]^{a-1}\left\{1-\frac{\left[\alpha(1+\lambda) e^{\left.-\left(\frac{\theta}{x}\right)^{\beta}-(\alpha \lambda+\alpha-1) e^{-2\left(\frac{\theta}{x}\right)^{\beta}}\right]^{a}}\right.}{\left[\alpha+(1-\alpha) e^{-\left(\frac{\theta}{x}\right)^{\beta}}\right]^{2 a}}\right\} .
\end{aligned}
$$

A physical interpretation of Equation (4) is possible when $a$ and $b$ are positive integers. Suppose a system is made up of $b$ independent components in series and that each component is made up of $a$ independent subcomponents in parallel. So, the system fails if any of the $b$ components fail and each component fails if all of its asubcomponents fail. 
If the sub-component lifetimes have a common $\mathrm{Kw}-\mathrm{TMOF}$ cumulative function, then the lifetime of the entire system will follow the Kw-TMOF distribution (4).

From another view; suppose a system consists of $b$ independent sub-systems functioning independently at a given time and that each sub-system consists of aindependent parallel components. Suppose too that each component consists of two units. If the two units are connected in series then the overall system will have Kw-TMOF distribution with $\lambda=1$ whereas if the components are parallel then the overall system will have Kw-TMOF distribution with $\lambda=-1$.

Furthermore, we can interpret the system from the redundancy view. Redundancy is a common method to increase reliability in an engineering design. Barlow and Proschan (1981) indicate that, if we want to increase the reliable of a given system, then redundancy at a component level is more effective than redundancy at a system level. That is, if all components of a system are available in duplicate, it is better to put these component pairs in parallel than it is to build two identical systems and place the systems in parallel.

The proposed Kw-TMOF model is very flexible model that approaches to different distributions when its parameters are changed. The flexibility of the Kw-TMOF is explained in Table 1 where it has sixty two sub-models when their parameters are carefully chosen. The reliability function (rf), hazard rate function (hrf) and cumulative hazard rate function (chrf) are, respectively, given by

$$
\begin{aligned}
& R(x, \phi)=\left\{1-\left[\alpha(1+\lambda) e^{-\left(\frac{\theta}{x}\right)^{\beta}}-(\alpha \lambda+\alpha-1) e^{-2\left(\frac{\theta}{x}\right)^{\beta}}\right]^{a}\left[\alpha+(1-\alpha) e^{-\left(\frac{\theta}{x}\right)^{\beta}}\right]^{-2 \mathrm{a}}\right\}^{b}, \\
& h(x, \phi)=\frac{a b \alpha \beta \theta^{\beta} x^{-\beta-1} \alpha(1+\lambda) e^{-\left(\frac{\theta}{x}\right)^{\beta}}-(\alpha \lambda+\alpha+\lambda-1) e^{-2\left(\frac{\theta}{x}\right)^{\beta}}}{\left[\alpha+(1-\alpha) e^{-\left(\frac{\theta}{x}\right)^{\beta}}\right]^{2 a+1}\left\{\alpha(1+\lambda) e^{-\left(\frac{\theta}{x}\right)^{\beta}}-(\alpha \lambda+\alpha-1) e^{-2\left(\frac{\theta}{x}\right)^{\beta}}\right\}^{1-a}} \\
& \times\left\{1-\left[\alpha(1+\lambda) e^{-\left(\frac{\theta}{x}\right)^{\beta}}-(\alpha \lambda+\alpha-1) e^{-2\left(\frac{\theta}{x}\right)^{\beta}}\right]^{a}\left[\alpha+(1-\alpha) e^{-\left(\frac{\theta}{x}\right)^{\beta}}\right]^{-2 a}\right\}^{-1}
\end{aligned}
$$

and

$H(x, \phi)=-b \ln \left\{1-\left[\alpha(1+\lambda) e^{-\left(\frac{\theta}{x}\right)^{\beta}}-(\alpha \lambda+\alpha-1) e^{-2\left(\frac{\theta}{x}\right)^{\beta}}\right]^{a}\left[\alpha+(1-\alpha) e^{-\left(\frac{\theta}{x}\right)^{\beta}}\right]^{-2 a}\right\}$.

Figure 1 provides some plots of the Kw-TMOF density curves for different values of the parameters $\alpha, \beta, \theta, \lambda, a$ and $b$. Some plots of the hrf of the Kw-TMOF are displayed in Figure 2. 

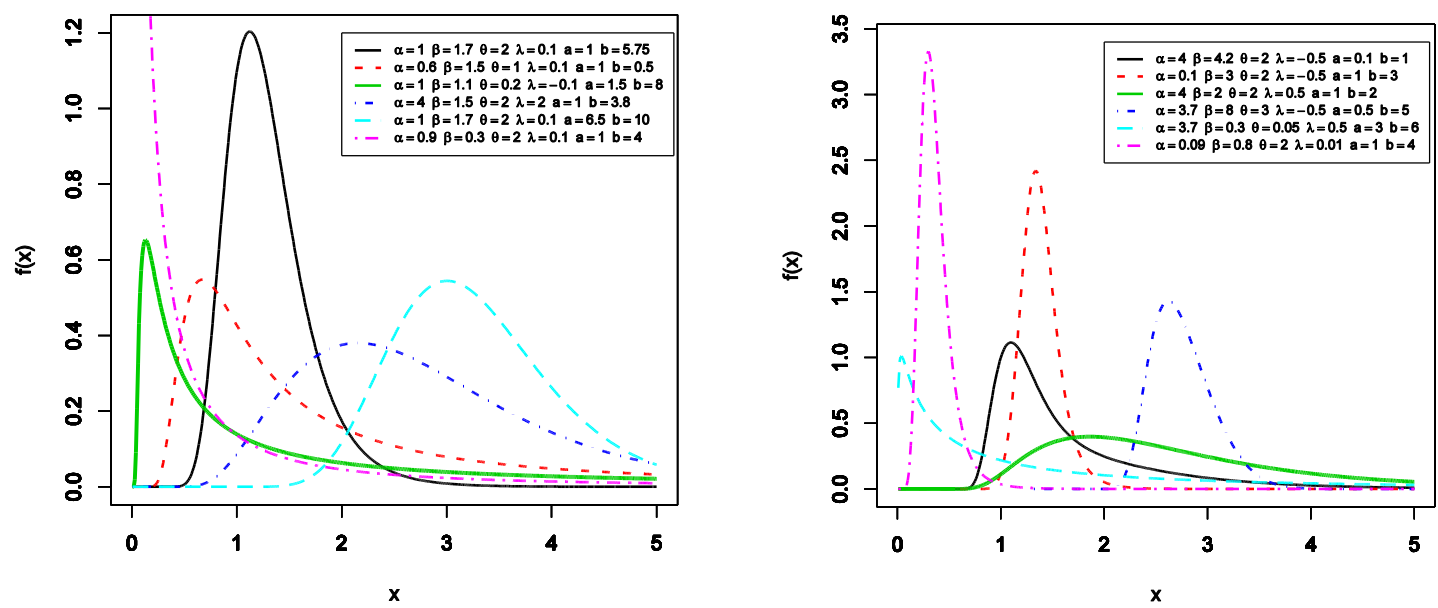

Figure 1: Plots of the Kw-TMOF density function for some parameter values.
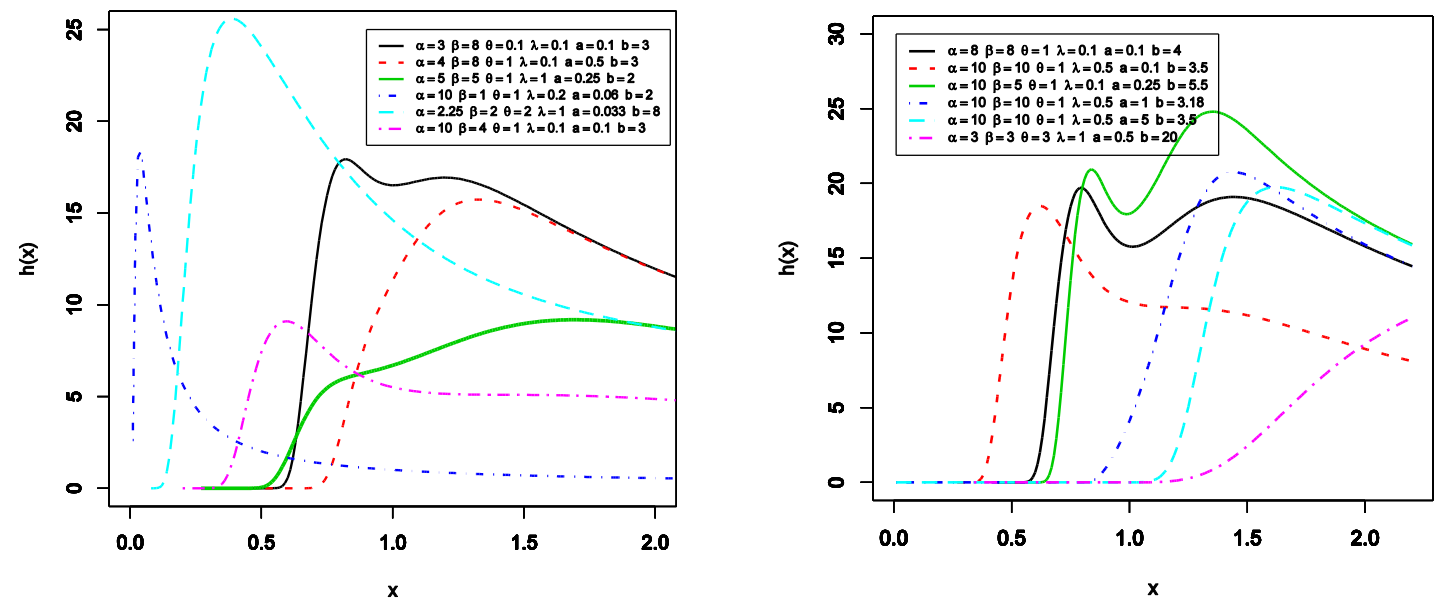

Figure 2: Plots of the Kw-TMOF hrf.

\section{Useful Expansions}

Expansions for Equations (3) and (4) can be derived using using the series expansion

$$
(1-z)^{k}=\sum_{j=0}^{\infty} \frac{(-1)^{j} \Gamma(k+1)}{j ! \Gamma(k-j+1)} z^{j},|z|<1, k>0 \text {. }
$$

The cdf of the Kw-TMOF in Equation (3) can be expressed in the mixture form

where

$$
F(x, \phi)=1-\sum_{j, i, k=0}^{\infty} s_{j, i . k} \exp \left[-(a j+k+i)\left(\frac{\theta}{x}\right)^{\beta}\right]
$$

$$
s_{j, i . k}=\frac{(-1)^{j+i} \Gamma(b+1) \Gamma(a j+1) \Gamma(2 a j+k)(\alpha \lambda+\alpha-1)^{i}}{j ! i ! k ! \alpha^{2 a j} \Gamma(2 a j) \Gamma(b-j+1) \Gamma(a j-i+1)(\alpha \lambda+\alpha)^{-a j+i}}\left(1-\frac{1}{\alpha}\right)^{k} .
$$

The pdf of the Kw-TMOF in (4) can be expressed in the mixture form

$$
f(x, \phi)=\beta \theta^{\beta} x^{-(\beta+1)} \sum_{j, i, k, w=0}^{\infty} b_{j, i, k, w} \exp \left[-(a j+a+i+k+w)\left(\frac{\theta}{x}\right)^{\beta}\right]
$$


Table 1: Sub-models of the Kw-TMOF distribution

\begin{tabular}{|c|c|c|c|c|c|c|c|c|}
\hline No. & Distribution & $\alpha$ & $\beta$ & $\theta$ & $\lambda$ & $a$ & $b$ & Author \\
\hline 1 & KTF & 1 & $\beta$ & $\theta$ & $\lambda$ & $a$ & $b$ & New \\
\hline 2 & KTMOIE & $\alpha$ & 1 & $\theta$ & $\lambda$ & $a$ & $b$ & New \\
\hline 3 & KTMOIR & $\alpha$ & 2 & $\theta$ & $\lambda$ & $a$ & $b$ & New \\
\hline 4 & KTMOGIW & $\alpha$ & $\beta$ & $q c^{1 / \beta}$ & $\lambda$ & $a$ & $b$ & New \\
\hline 5 & KTMOGuII & $\alpha$ & $\beta$ & $p=\theta^{\beta}$ & $\lambda$ & $a$ & $b$ & New \\
\hline 6 & KTMOIW & $\alpha$ & $\beta$ & $c^{1 / \beta}$ & $\lambda$ & $a$ & $b$ & New \\
\hline 7 & KTGIW & 1 & $\beta$ & $q c^{1 / \beta}$ & $\lambda$ & $a$ & $b$ & New \\
\hline 8 & KTGuII & 1 & $\beta$ & $p=\theta^{\beta}$ & $\lambda$ & $a$ & $b$ & New \\
\hline 9 & KTIW & 1 & $\beta$ & $c^{1 / \beta}$ & $\lambda$ & $a$ & $b$ & New \\
\hline 10 & KTIE & 1 & 1 & $\theta$ & $\lambda$ & $a$ & $b$ & New \\
\hline 11 & KTIR & 1 & 2 & $\theta$ & $\lambda$ & $a$ & $b$ & New \\
\hline 12 & $\mathrm{KF}$ & 1 & $\beta$ & $\theta$ & 0 & $a$ & $b$ & Mead and Abd-Eltawab (2014) \\
\hline 13 & KMOF & $\alpha$ & $\beta$ & $\theta$ & 0 & $a$ & $b$ & Afify et al. (2016) \\
\hline 14 & KMOIE & $\alpha$ & 1 & $\theta$ & 0 & $a$ & $b$ & -- \\
\hline 15 & KMOIR & $\alpha$ & 2 & $\theta$ & 0 & $a$ & $b$ & -- \\
\hline 16 & KMOGIW & $\alpha$ & $\beta$ & $q c^{1 / \beta}$ & 0 & $a$ & $b$ & New \\
\hline 17 & KMOGuII & $\alpha$ & $\beta$ & $p=\theta^{\beta}$ & 0 & $a$ & $b$ & New \\
\hline 18 & KMOIW & $\alpha$ & $\beta$ & $c^{1 / \beta}$ & 0 & $a$ & $b$ & New \\
\hline 19 & KGIW & 1 & $\beta$ & $q c^{1 / \beta}$ & 0 & $a$ & $b$ & Afify et al. (2016) \\
\hline 20 & KGuII & 1 & $\beta$ & $p=\theta^{\beta}$ & 0 & $a$ & $b$ & Afify et al. (2016) \\
\hline 21 & KIW & 1 & $\beta$ & $c^{1 / \beta}$ & 0 & $a$ & $b$ & Shahbaz et al. (2012) \\
\hline 22 & KIE & 1 & 1 & $\theta$ & 0 & $a$ & $b$ & Mead and Abd-Eltawab (2014) \\
\hline 23 & KIR & 1 & 2 & $\theta$ & 0 & $a$ & $b$ & Mead and Abd-Eltawab (2014) \\
\hline 24 & TMOF & $\alpha$ & $\beta$ & $\theta$ & $\lambda$ & 1 & 1 & Afify et al. (2015) \\
\hline 25 & TMOIE & $\alpha$ & 1 & $\theta$ & $\lambda$ & 1 & 1 & -- \\
\hline 26 & TMOIR & $\alpha$ & 2 & $\theta$ & $\lambda$ & 1 & 1 & -- \\
\hline 27 & TMOGIW & $\alpha$ & $\beta$ & $q c^{1 / \beta}$ & $\lambda$ & 1 & 1 & New \\
\hline 28 & TMOGuII & $\alpha$ & $\beta$ & $p=\theta^{\beta}$ & $\lambda$ & 1 & 1 & New \\
\hline 29 & TMOIW & $\alpha$ & $\beta$ & $c^{1 / \beta}$ & $\lambda$ & 1 & 1 & New \\
\hline 30 & TEGIW & 1 & $\beta$ & $q c^{1 / \beta}$ & $\lambda$ & 1 & $b$ & New \\
\hline 31 & TEGuII & 1 & $\beta$ & $p=\theta^{\beta}$ & $\lambda$ & 1 & $b$ & New \\
\hline 32 & TEIW & 1 & $\beta$ & $c^{1 / \beta}$ & $\lambda$ & 1 & $b$ & New \\
\hline 33 & TGIW & 1 & $\beta$ & $q c^{1 / \beta}$ & $\lambda$ & 1 & 1 & Merovci et al. (2013) \\
\hline 34 & TGuII & 1 & $\beta$ & $p=\theta^{\beta}$ & $\lambda$ & 1 & 1 & New \\
\hline 35 & TIW & 1 & $\beta$ & $c^{1 / \beta}$ & $\lambda$ & 1 & 1 & Khan et al. (2013) \\
\hline 36 & MOFD & $\alpha$ & $\beta$ & $\theta$ & 0 & 1 & 1 & Krishna et al. (2013) \\
\hline 37 & MOIE & $\alpha$ & 1 & $\theta$ & 0 & 1 & 1 & -- \\
\hline 38 & MOIR & $\alpha$ & 2 & $\theta$ & 0 & 1 & 1 & -- \\
\hline 39 & MOGIW & $\alpha$ & $\beta$ & $q c^{1 / \beta}$ & 0 & 1 & 1 & New \\
\hline 40 & MOGuII & $\alpha$ & $\beta$ & $p=\theta^{\beta}$ & 0 & 1 & 1 & New \\
\hline 41 & MOIW & $\alpha$ & $\beta$ & $c^{1 / \beta}$ & 0 & 1 & 1 & New \\
\hline 42 & TEF & 1 & $\beta$ & $\theta$ & $\lambda$ & 1 & $b$ & Elbatal et al. (2014) \\
\hline 43 & TEIE & 1 & 1 & $\theta$ & $\lambda$ & 1 & $b$ & -- \\
\hline 44 & TEIR & 1 & 2 & $\theta$ & $\lambda$ & 1 & $b$ & -- \\
\hline 45 & $\mathrm{TF}$ & 1 & $\beta$ & $\theta$ & $\lambda$ & 1 & 1 & Mahmoud and Mandouh (2013) \\
\hline 46 & TIE & 1 & 1 & $\theta$ & $\lambda$ & 1 & 1 & Oguntunde and Adejumo (2015) \\
\hline 47 & TIR & 1 & 2 & $\theta$ & $\lambda$ & 1 & 1 & Ahmad et al. (2014) \\
\hline 48 & $\mathrm{EF}$ & 1 & $\beta$ & $\theta$ & 0 & 1 & $b$ & Nadarajah and Kotz (2003) \\
\hline 49 & EIE & 1 & 1 & $\theta$ & 0 & 1 & $b$ & -- \\
\hline 50 & EIR & 1 & 2 & $\theta$ & 0 & 1 & $b$ & -- \\
\hline 51 & TGF & 1 & $\beta$ & $\theta$ & $\lambda$ & $a$ & 1 & New \\
\hline 52 & TGIE & 1 & 1 & $\theta$ & $\lambda$ & $a$ & 1 & New \\
\hline 53 & TGIR & 1 & 2 & $\theta$ & $\lambda$ & $a$ & 1 & New \\
\hline 54 & GF & 1 & $\beta$ & $\theta$ & 0 & $a$ & 1 & New \\
\hline 55 & GIE & 1 & 1 & $\theta$ & 0 & $a$ & 1 & New \\
\hline 56 & GIR & 1 & 2 & $\theta$ & 0 & $a$ & 1 & New \\
\hline
\end{tabular}




\begin{tabular}{ccccccccc}
\hline No. & Distribution & $\boldsymbol{\alpha}$ & $\boldsymbol{\beta}$ & $\boldsymbol{\theta}$ & $\boldsymbol{\lambda}$ & $\boldsymbol{a}$ & $\boldsymbol{b}$ & Author \\
\hline 57 & GIW & 1 & $\beta$ & $q c^{1 / \beta}$ & 0 & 1 & 1 & de Gusmão et al. (2011) \\
58 & GuII & 1 & $\beta$ & $p=\theta^{\beta}$ & 0 & 1 & 1 & Gumbel (1958) \\
59 & IW & 1 & $\beta$ & $c^{1 / \beta}$ & 0 & 1 & 1 & Keller et al. (1982) \\
60 & F & 1 & $\beta$ & $\theta$ & 0 & 1 & 1 & Fréchet (1924) \\
61 & IE & 1 & 1 & $\theta$ & 0 & 1 & 1 & Keller and Kamath (1982) \\
62 & IR & 1 & 2 & $\theta$ & 0 & 1 & 1 & Trayer (1964) \\
\hline
\end{tabular}

where

$$
b_{j, i, k, w}=\frac{a b(-1)^{j+i+w} \Gamma(b) \Gamma(a j+a) \Gamma(2 a j+2 a+k+1)\left(1-\frac{1}{\alpha}\right)^{k}(\alpha \lambda+\alpha)^{a(j+1)-i-w}}{j ! i ! k ! w ! \Gamma(b-j) \Gamma(a j+a-i) \Gamma(2 a j+2 a+1) \Gamma(2-w) \alpha^{2 a(j+1)}(\alpha \lambda+\alpha-1)^{-i}(\alpha \lambda+\alpha+\lambda-1)^{-w}} .
$$

The Kw-TMOF density function can be expressed as a mixture of Fréchet densities. Thus, some of its mathematical properties can be obtained directly from those properties of the Fréchet distribution. Therefore Equation (4) can be also expressed as

$$
f(x, \phi)=\sum_{j, i, k, w=0}^{\infty} \frac{b_{j, i, k, w}}{(a j+a+i+k+w)} g\left(x ; \beta, \theta^{*}\right),
$$

where $g\left(x ; \beta, \theta^{*}\right)$ denotes to the Fréchet pdf where $\theta^{*}=\theta(a j+a+i+k+w)^{1 / \beta}$.

\section{Properties}

\section{Quantile Function}

The quantile function (qf) of $X$ is obtained by inverting (3) as

$$
Q(u)=\theta\left\{\ln \left(\frac{\left.\kappa+\sqrt{\alpha^{2}\left(\lambda^{2}+2 \lambda+1\right)-4 \alpha^{2} \lambda \sqrt[a]{1-\sqrt[b]{1-u}}}\right)}{2 \alpha^{2} \sqrt[a]{1-\sqrt[b]{1-u}}}\right)\right\}^{-1 / \beta} \quad, 0 \leq u \leq 1,
$$

where $\kappa=\alpha(\lambda+1)-2 \alpha(1-\alpha) \sqrt[a]{1-\sqrt[b]{1-u}}$

Simulating the $\mathrm{Kw}-\mathrm{TMOF}$ random variable is straightforward. If $U$ is a uniform variate on the unit interval $(0,1)$, then the random variable $X=Q(U)$ follows (4), i.e. $X: \mathrm{Kw}$ TMOF $(\alpha, \beta, \theta, \lambda, a, b)$.

\section{Ordinary and Incomplete Moments}

Therth moment, denoted by $\mu_{r}^{\prime}$, of $X$ (for $r<\beta$ ) is given as by

$$
\mu_{r}^{\prime}=E\left(X^{r}\right)=\frac{\theta^{r} \sum_{j, i, k, w=0}^{\infty} b_{j, i, k, w}}{(a j+a+i+k+w)^{1-(\mathrm{r} / \beta)}} \Gamma\left(1-\frac{r}{\beta}\right) .
$$

Sitting $r=1$, we get the mean of $X$. The skewness and kurtosis measures can be calculated from the ordinary moments using well-known relationships. 
Corollary 1. Using the relation between the central moments and non-centeral moments, we can obtain the $n$th central moment, denoted by $M_{n}$, of a Kw-TMOF random variable as follows

$$
M_{n}=E(X-\mu)^{n}=\sum_{r=0}^{n}\left(\begin{array}{l}
n \\
r
\end{array}\right)(-\mu)^{n-r} E\left(X^{r}\right),
$$

where $E\left(X^{r}\right)$ is the on-central moments of the $\operatorname{Kw}$-TMOF $(\alpha, \beta, \theta, \lambda, a, b, x)$. Therefore the $n$th central moments of the $\mathrm{Kw}-\mathrm{TMOF}(\alpha, \beta, \theta, a, b, x)$, if $r<\beta$, is given by

$$
M_{n}=\sum_{r=0}^{n}\left(\begin{array}{l}
n \\
r
\end{array}\right)(-\mu)^{n-r} \frac{\theta^{r} \sum_{j, i, k, w=0}^{\infty} b_{j, i, k, w}}{(a j+a+i+k+w)^{1-(\mathrm{r} / \beta)}} \Gamma\left(1-\frac{r}{\beta}\right) .
$$

The moment generating function $(m g f)$ of $X$, say $M_{X}(t)=E\left(e^{t X}\right)$, (for $\left.r<\beta\right)$ is given by

$$
M_{X}(t)=\sum_{r=0}^{\infty} \frac{(t \theta)^{r} \sum_{j, i, k, w=0}^{\infty} b_{j, i, k, w}}{r !(a j+a+i+k+w)^{1-(\mathrm{r} / \beta)}} \Gamma\left(1-\frac{r}{\beta}\right) .
$$

The $s$ th incomplete moments, denoted by $\varphi_{s}(t)$,of $X$ is given by

$$
\varphi_{s}(t)=\int_{0}^{t} x^{s} f(x) d x
$$

Using Equation (6) and the lower incomplete gamma function, if $s<\beta$, we obtain

$\varphi_{s}(t)=\sum_{j, i, k, w=0}^{\infty} \frac{b_{j, i, k, w} \theta^{s}}{(a j+a+i+k+w)^{1-(s / \beta)}} \gamma\left(1-\frac{s}{\beta},(a j+a+i+k+w)\left(\frac{\theta}{t}\right)^{\beta}\right)$.

The first incomplete moment of $X$,denoted by, $\varphi_{1}(t)$, is immediately calculated from Equation (8) by setting $s=1$.

\section{Rényi and $\eta$-Entropies}

The Rényi entropy of $X$ represents a measure of variation of the uncertainty. The Rényi entropy is defined by

$$
I_{\eta}(X)=\frac{1}{1-\eta} \log \int_{-\infty}^{\infty} f^{\eta}(x) d x, \eta>0 \text { and } \eta \neq 1 .
$$

Therefore, the Rényi entropy of a random variable $X$ which follows the Kw-TMOF $(x, \phi)$ is given by

$$
I_{\eta}(X)=\frac{\log \left(\beta a b \theta^{\beta}\right)^{\eta}}{1-\eta} \sum_{j, i, k, w=0}^{\infty} \mathrm{t}_{j, i, k, w} \underbrace{\int_{0}^{\infty} x^{-\eta(1+\beta)} \exp \left[-(-a \eta-a j-\eta+i+w)\left(\frac{\theta}{x}\right)^{\beta}\right] d x}_{A}
$$


Where

$$
\begin{aligned}
\mathrm{t}_{j, i, k, w} & =\frac{(-1)^{j+i+w} \Gamma(\eta b-\eta+1) \Gamma(a j+a \eta-\eta+1) \Gamma(2 a j+2 a \eta+\eta+k)}{j ! i ! k ! w ! \Gamma(\eta b-(\eta+j)+1) \Gamma(a j+a \eta-\eta-i+1) \Gamma(2 a j+2 a \eta+\eta)} \\
& \times \frac{\Gamma(\eta+1)(a b)^{\eta}(\alpha \lambda+\alpha)^{a j+a-i-w}\left(1-\frac{1}{\alpha}\right)^{-2 a j+2 a \eta+\eta}}{\Gamma(\eta+1-w) \alpha^{2 a(\eta+j)}(\alpha \lambda+\alpha-1)^{-i}(\alpha \lambda+\alpha+\lambda-1)^{-w}}
\end{aligned}
$$

But

and then

$$
A=\frac{\theta^{1-\eta(1+\beta)}}{\beta(-a \eta-a j-\eta+i+w)^{-(1-\lambda(1+\beta)) / \beta}} \Gamma\left(\frac{\eta(1+\beta)-1}{\beta}\right) .
$$

$$
I_{\lambda}(X)=\frac{1}{1-\eta} \log \left\{\left(\frac{\theta}{\beta}\right)^{1-\eta} \sum_{j, i, k, w=0}^{\infty} \frac{\mathrm{t}_{j, i, k, w}}{(-a \eta-a j-\eta+i+w)^{p}} \Gamma(p)\right\} .
$$

The $\eta$-entropy, say $H_{\eta}(X)$, is defined by

$$
\begin{aligned}
& H_{\eta}(X)=\frac{1}{\eta-1} \log \left\{1-\int_{-\infty}^{\infty} f^{\eta}(x) d x\right\}, \eta>0 \text { and } \eta \neq 1 . \\
& H_{\eta}(X)=\frac{1}{\eta-1} \log \left\{1-\left(\frac{\theta}{\beta}\right)^{1-\eta} \sum_{j, i, k, w=0}^{\infty} \frac{\mathrm{t}_{j, i, k, w}}{(-a \eta-a j-\eta+i+w)^{p}} \Gamma(p)\right\},
\end{aligned}
$$

where $p=(\lambda \beta+\lambda-1) / \beta$.

\section{Moments of Residual and Reversed Residual Lifes}

The $n$th moments of residual life, denoted by $m_{n}(t)=E\left((X-t)^{n} \mid X>t\right), n=1,2,3, \ldots$, is defined by $m_{n}(t)=\frac{1}{1-F(t)} \int_{t}^{\infty}(x-t)^{n} d F(x)$. Therefore, the $n$th moments of the residual life of $X$ given that $r<\beta$, is given by

$m_{n}(t)=\frac{1}{R(t)} \sum_{r=0}^{n} \frac{(-1)^{n-r} \Gamma(n+1) t^{n-r} \theta^{r} \sum_{j, i, k, w=0}^{\infty} b_{j, i, k, w}}{r ! \Gamma(n-r+1)(a j+a+i+k+w)^{1-(r / \beta)}} \gamma\left(1-\frac{r}{\beta},(a j+a+i+k+w)\left(\frac{\theta}{t}\right)^{\beta}\right)$.

Here we can use the upper incomplete gamma function defined by $\gamma(a, b)=$ $\int_{b}^{\infty} y^{a-1} e^{-y} d y$. Another interesting function is the mean residual life function (MRL) or the life expectancy at age $\mathrm{t}$, defined by $m_{1}(x)=E((X-x) \mid X>x)$, and it represents the expected additional life length for a unit which is alive at age $x$. The MRL of the KwTMOF distribution can be obtained by setting $n=1$ in the last equation. The $n$th moments of the reversed residual life, denoted by $M_{n}(t)=E\left((t-X)^{n} \mid X \leq t\right), t>$ $0, n=1,2,3, \ldots$, is given by $M_{n}(t)=\frac{1}{F(t)} \int_{0}^{t}(t-x)^{n} d F(x)$. Therefore, The $n$th moments of the reversed residual life of a Kw-TMOF $(\alpha, \beta, \lambda, \eta)$, given that $r<\beta$, is given by

$M_{n}(t)=\frac{1}{F(t)} \sum_{r=0}^{n} \frac{(-1)^{r} \Gamma(n+1) t^{n-r} \theta^{r} \sum_{j, i, k, w=0}^{\infty} b_{j, i, k, w}}{r ! \Gamma(n-r+1)(a j+a+i+k+w)^{1-(\mathrm{r} / \beta)}} \gamma\left(1-\frac{r}{\beta},(a j+a+i+k+w)\left(\frac{\theta}{t}\right)^{\beta}\right)$. 
Here we can use the lower incomplete gamma function defined by $\gamma(a, b)=$ $\int_{0}^{b} y^{a-1} e^{-y} d y$. The mean inactivity time (MIT) or mean waiting time (MWT) also called mean reversed residual life function, defined by $M_{1}(t)=E((t-X) \mid X \leq t)$, and it represents the waiting time elapsed since the failure of an itemon condition that this failure had occurred in $(0, x)$. The MRRL of the Kw-TMOF distribution can be obtained by setting $n=1$.

\section{Order Statistics}

The order statistics and their moments have great importance in many statistical problems and they have many applications in reliability analysis and life testing. The order statistics arise in the study of reliability of a system. The order statistics can represent the lifetimes of units or components of a reliability system. Let $X_{1}, X_{2}, \ldots, X_{n}$ be a random sample of size $n$ from the $\operatorname{Kw}-\mathrm{TMOF}(\phi, x)$ with cdf and pdf as in (3) and (4), respectively. Let $X_{(1)}, X_{(2)}, \ldots, X_{(n)}$ be the corresponding order statistics. Then the pdf of $j$ th order statistics, say $Y=X_{(j: n)}, 1 \leq j \leq n$,denoted by $f_{Y}(x)$,is given by

$$
\begin{aligned}
& f_{Y}(x)=\frac{n ! a b \alpha \beta \theta^{\beta} x^{-(\beta+1)}}{(j-1) !(n-j) !} \frac{\alpha(1+\lambda) \exp \left[-\left(\frac{\theta}{x}\right)^{\beta}\right]-(\alpha \lambda+\alpha+\lambda-1) \exp \left[-2\left(\frac{\theta}{x}\right)^{\beta}\right]}{\left\{\alpha+(1-\alpha) \exp \left[-\left(\frac{\theta}{x}\right)^{\beta}\right]\right\}^{2 a+1}\left\{\frac{\alpha(1+\lambda)}{\exp \left[\left(\frac{\theta}{x}\right)^{\beta}\right]}-\frac{(\alpha \lambda+\alpha-1)}{\exp \left[2\left(\frac{\theta}{x}\right)^{\beta}\right]}\right\}^{1-a}} \\
& \times\left\{1-\left(\frac{\alpha(1+\lambda) \exp \left[-\left(\frac{\theta}{x}\right)^{\beta}\right]-(\alpha \lambda+\alpha-1) \exp \left[-2\left(\frac{\theta}{x}\right)^{\beta}\right]}{\left(\alpha+(1-\alpha) \exp \left[-\left(\frac{\theta}{x}\right)^{\beta}\right]\right)^{2}}\right)^{a}\right\}^{b(n-j+1)-1} \\
& \times\left\{1-\left[1-\left(\frac{\alpha(1+\lambda) \exp \left[-\left(\frac{\theta}{x}\right)^{\beta}\right]-(\alpha \lambda+\alpha-1) \exp \left[-2\left(\frac{\theta}{x}\right)^{\beta}\right]}{\left(\alpha+(1-\alpha) \exp \left[-\left(\frac{\theta}{x}\right)^{\beta}\right]\right)^{2}}\right)^{a}\right\}^{b-1} .\right.
\end{aligned}
$$

The pdf of $Y$ in (9) can be expressed as a mixture of Fréchet densities as

$$
f_{Y}(x)=\sum_{r, i, l, h, w=0}^{\infty} \xi_{r, i, l, h, w} g\left(x ; \beta, \theta(a i+a+i+l+h+w)^{1 / \beta}\right),
$$

where $g\left(x ; \beta, \theta(a i+a+i+l+h+w)^{1 / \beta}\right)$ denotes to the Fréchet pdf with parameters $\beta, \theta(a i+a+i+l+h+w)^{1 / \beta}$ and

$\xi_{j, i, k, w}=\frac{n ! a b(-1)^{r+i+l+h} \Gamma(2 a i+2 a+1+w) \alpha^{-2 a(i+1)}(\alpha \lambda+\alpha-1)^{h}\left(\begin{array}{c}b(n+r-j+1)-1 \\ i\end{array}\right)\left(\begin{array}{c}j-1 \\ r\end{array}\right)\left(\begin{array}{l}a(i+1)-1 \\ h\end{array}\right)\left(1-\frac{1}{\alpha}\right)^{w}}{(j-1) !(n-j) ! l ! w ! \Gamma(2-l) \Gamma(2 a i+2 a+1)(a i+a+i+l+h+w)(\alpha \lambda+\alpha+\lambda-1)^{-l}(\alpha \lambda+\alpha)^{-(a i+a-l-h)}}$.

Therefore the qth moment of $Y$ can be expressed as

$$
E\left(Y^{q}\right)=\sum_{r, i, l, h, w=0}^{\infty} \xi_{r, i, l, h, w} E\left(Y_{\beta, \theta(a i+a+i+l+h+w)^{1 / \beta}}^{q}\right),
$$

where $Y_{\beta, \theta(a i+a+i+l+h+w)^{1 / \beta}}$ : Fréchet $\left(\beta, \theta(a i+a+i+l+h+w)^{1 / \beta}\right)$. 


\section{Characterization}

Characterizations of distributions is an important research area which has recently attracted the attention of many researchers. This section deals with a characterization of $\mathrm{Kw}-\mathrm{TMOF}$ distribution. This characterization is in terms of a truncated moment of a function of the random variable. Our characterization result employs a special case of a theorem due to Glänzel (1987), see Theorem 1 below. Note that the result holds also when the interval $H$ is not closed. Moreover, it could be also applied when the cdf $F$ does not have a closed form. As shown in Glänzel (1990), this characterization is stable in the sense of weak convergence.

Theorem 1. Let $(\Omega, \mathcal{F}, \mathbf{P})$ be a given probability space and let $H=[d, e]$ be an interval for some $d<e \quad(d=-\infty, e=\infty$ mightaswellbeallowed). Let $X: \Omega \rightarrow H$ be a continuous random variable with the distribution function $F$ and let $g$ be a real function defined on $H$ such that

$$
\mathbf{E}[g(X) \mid X \geq x]=\xi(x), \quad x \in H,
$$

is defined with some real function $\xi$. Assume that $g \in C^{1}(H), \xi \in C^{2}(H)$ and $F$ is twice continuously differentiable and strictly monotone function on the set $H$. Finally, assume that the equation $\xi=g$ has no real solution in the interior of $H$. Then $F$ is uniquely determined by the functions $g$ and $\xi$, particularly

$$
F(x)=\int_{a}^{x} C\left|\frac{\xi^{\prime}(u)}{\xi(u)-g(u)}\right| \exp (-s(u)) d u,
$$

where the function $s$ is a solution of the differential equation $s^{\prime}=\frac{\xi^{\prime}}{\xi-g}$ and $C$ is the normalization constant, such that $\int_{H} d F=1$.

Here is our first characterization.

Proposition 1. Let $X: \Omega \rightarrow(0, \infty)$ be a continuous random variable and let

$$
g(x)=\left\{1-\frac{\left[\alpha(1+\lambda) e^{-(\theta / x)^{\beta}}-(\alpha \lambda+\alpha-1) e^{-2(\theta / x)^{\beta}}\right]^{a-1}}{\left[\alpha+(1-\alpha) e^{-(\theta / x)^{\beta}}\right]^{2 a}}\right\}, \text { for } x>0 .
$$

The random variable $X$ belongs to Kw-TMOF family (4) if and only if the function $\xi$ defined in Theorem 1 has the form

$$
\xi(x)=\frac{b}{b+1}\left\{1-\left(\frac{\alpha(1+\lambda) e^{-(\theta / x)^{\beta}}-(\alpha \lambda+\alpha-1) e^{-2(\theta / x)^{\beta}}}{\left[\alpha+(1-\alpha) e^{-(\theta / x)^{\beta}}\right]^{2}}\right)^{a}\right\}, x>0 .
$$

Proof. Let $X$ be a random variable with pdf (4), then

$$
\begin{aligned}
& (1-F(x)) E[g(x) \mid X \geq x] \\
& =\int_{x}^{\infty} f(u)\left\{1-\frac{\left[\alpha(1+\lambda) e^{-(\theta / u)^{\beta}}-(\alpha \lambda+\alpha-1) e^{-2(\theta / u)^{\beta}}\right]^{a-1}}{\left[\alpha+(1-\alpha) e^{-(\theta / u)^{\beta}}\right]^{2 a}}\right\} d u \\
& =\frac{b}{b+1}\left\{1-\left(\frac{\alpha(1+\lambda) e^{-(\theta / x)^{\beta}}-(\alpha \lambda+\alpha-1) e^{-2(\theta / x)^{\beta}}}{\left[\alpha+(1-\alpha) e^{-(\theta / x)^{\beta}}\right]^{2}}\right)^{a+1}, x>0\right.
\end{aligned}
$$


and

$$
\begin{aligned}
\xi(x)-g(x) & =-\frac{1}{b+1}\left\{1-\left(\frac{\alpha(1+\lambda) e^{-(\theta / x)^{\beta}}-(\alpha \lambda+\alpha-1) e^{-2(\theta / x)^{\beta}}}{\left[\alpha+(1-\alpha) e^{-(\theta / x)^{\beta}}\right]^{2}}\right)^{a}\right\} \\
& <0, \text { for } x>0 .
\end{aligned}
$$

Conversely, if $\xi$ is given as above, then

$$
\begin{gathered}
s^{\prime}(x)=\frac{\xi^{\prime}(x)}{\xi(x)-g(x)}=\frac{b a \alpha \beta \theta^{\beta} x^{-\beta-1}\left[\alpha(1+\lambda) e^{-(\theta / u)^{\beta}}-(\alpha \lambda+\alpha+\lambda-1) e^{-2(\theta / u)^{\beta}}\right]}{\left[\alpha+(1-\alpha) e^{-(\theta / u)^{\beta}}\right]^{2 a+1}\left\{1-\left(\frac{\alpha(1+\lambda) e^{-(\theta / x)^{\beta}}-(\alpha \lambda+\alpha-1) e^{-2(\theta / x) \beta}}{\left[\alpha+(1-\alpha) e^{-(\theta / x)}\right]^{2}}\right]^{a}\right)} \times \\
{\left[\alpha(1+\lambda) e^{-(\theta / u)^{\beta}}-(\alpha \lambda+\alpha-1) e^{-2(\theta / u)^{\beta}}\right]^{a-1}}
\end{gathered}
$$

and hence

$$
s(x)=-b \log \left\{1-\left(\frac{\alpha(1+\lambda) e^{-(\theta / x)^{\beta}}-(\alpha \lambda+\alpha-1) e^{-2(\theta / x)^{\beta}}}{\left[\alpha+(1-\alpha) e^{-(\theta / x)^{\beta}}\right]^{2}}\right)^{a}, \quad x>0 .\right.
$$

Now, in view of Theorem $1, X$ has density (4).

Corollary 2. Let $X: \Omega \rightarrow(0, \infty)$ be a continuous random variable. The pdf of $X$ is $(4)$ if and only if there exist functions $g$ and $\xi$ defined in Theorem 1 satisfying the differential equation

$$
\begin{aligned}
& \frac{\xi^{\prime}(x)}{\xi(x)-g(x)}=\frac{\operatorname{ba\alpha } \beta \theta^{\beta} x^{-\beta-1}\left[\alpha(1+\lambda) e^{-(\theta / u)^{\beta}}-(\alpha \lambda+\alpha+\lambda-1) e^{-2(\theta / u)^{\beta}}\right]}{\left[\alpha+(1-\alpha) e^{-(\theta / u)^{\beta}}\right]^{2 a+1}\left\{1-\left(\frac{\alpha(1+\lambda) e^{-(\theta / x)^{\beta}}-(\alpha \lambda+\alpha-1) e^{-2(\theta / x)^{\beta}}}{\left[\alpha+(1-\alpha) e^{-(\theta / x)^{\beta}}\right]^{2}}\right)\right.} \times \\
& {\left[\alpha(1+\lambda) e^{-(\theta / u)^{\beta}}-(\alpha \lambda+\alpha-1) e^{-2(\theta / u)^{\beta}}\right]^{a-1} .}
\end{aligned}
$$

The general solution of the differential equation in Corollary 2 is

$$
\begin{aligned}
& \xi(x)=\left\{1-\left(\frac{\alpha(1+\lambda) e^{-(\theta / x)^{\beta}}-(\alpha \lambda+\alpha-1) e^{-2(\theta / x)^{\beta}}}{\left[\alpha+(1-\alpha) e^{-(\theta / x)^{\beta}}\right]^{2}}\right)^{a}\right\}^{-b} \\
& -\int \frac{b a \alpha \beta \theta^{\beta} x^{-\beta-1}\left[\alpha(1+\lambda) e^{-(\theta / u)^{\beta}}-(\alpha \lambda+\alpha+\lambda-1) e^{-2(\theta / u)^{\beta}}\right]}{\left[\alpha+(1-\alpha) e^{-(\theta / u)^{\beta}}\right]^{2 a+1}} \times \\
& {\left[\begin{array}{l}
\left.\alpha(1+\lambda) e^{-(\theta / u)^{\beta}}-(\alpha \lambda+\alpha-1) e^{-2(\theta / u)^{\beta}}\right]^{a-1} \times \\
\left\{1-\left(\frac{\alpha(1+\lambda) e^{-(\theta / x)^{\beta}}-(\alpha \lambda+\alpha-1) e^{-2(\theta / x)^{\beta}}}{\left[\alpha+(1-\alpha) e^{-(\theta / x)^{\beta}}\right]^{2}}\right)^{a-1} g(x) d x+D\right.
\end{array}\right],}
\end{aligned}
$$

where $D$ is a constant. Note that a set of functions satisfying the differential Equation (10) is given in Proposition 1 with $D=0$. However, it should be also noted that there are other pairs $(g, \xi)$ satisfying the conditions of Theorem 1 . 


\section{Estimation}

The maximum likelihood estimators (MLEs) for the parameters of the Kw-TMOF is discussed in this section. Let $x_{1}, \ldots, x_{n}$ be a random sample of this distribution with unknown parameter vector $\phi=(\alpha, \beta, \theta, \lambda, a, b)^{T}$. Then, the log-likelihood function for $\phi, \ell=\ell(\phi)$, is:

$$
\begin{aligned}
\ell & =n(\ln a+\ln b+\ln \alpha+\ln \beta+\beta \ln \theta)-(\beta+1) \sum_{i=1}^{\mathrm{n}} \ln x_{i}-\theta^{\beta} \sum_{i=1}^{\mathrm{n}} x_{i}^{\beta} \\
& +\sum_{i=1}^{\mathrm{n}} \ln l_{i}+(a-1) \sum_{i=1}^{\mathrm{n}} \ln k_{i}-(2 a+1) \sum_{i=1}^{\mathrm{n}} \ln z_{i}+(b-1) \sum_{i=1}^{\mathrm{n}} \ln q_{i},
\end{aligned}
$$

where $l_{i}=\alpha(1+\lambda)-(\lambda+p) s_{i}, \quad k_{i}=\alpha(1+\lambda) s_{i}-p s_{i}^{2}, \quad p=\alpha \lambda+\alpha-1$,

$$
z_{i}=\alpha+(1-\alpha) s_{i}, q_{i}=1-\left(\frac{k_{i}}{z_{i}^{2}}\right)^{a} \text { and } s_{i}=\exp \left[-\left(\frac{\theta}{x_{i}}\right)^{\beta}\right] \text {. }
$$

The elements of score vector, $\mathbf{U}(\phi)=\frac{\partial \ell}{\partial \phi}=\left(\frac{\partial \ell}{\partial \alpha}, \frac{\partial \ell}{\partial \beta}, \frac{\partial \ell}{\partial \lambda}, \frac{\partial \ell}{\partial \theta}, \frac{\partial \ell}{\partial a}, \frac{\partial \ell}{\partial b}\right)^{T}$, are given by

$$
\begin{aligned}
& \frac{\partial \ell}{\partial \alpha}=\frac{n}{\alpha}+\sum_{i=1}^{\mathrm{n}} \frac{(1+\lambda)}{l_{i}\left(1-s_{i}\right)^{-1}}+(a-1) \sum_{i=1}^{\mathrm{n}} \frac{(1+\lambda)}{k_{i}\left(1-s_{i}\right)^{-1}} \\
& +(2 a+1) \sum_{i=1}^{\mathrm{n}} \frac{(1+\alpha) \ln \left(\frac{\theta}{x_{i}}\right)}{k_{i} s_{i}^{-1}\left(\frac{\theta}{x_{i}}\right)^{-1}}-(b-1) \sum_{i=1}^{\mathrm{n}} \frac{a l_{i}\left(1-q_{i}\right)^{\frac{a-1}{a}}}{z_{i}^{3} q_{i}}, \\
& \frac{\partial \ell}{\partial \beta}=n\left(\frac{1}{\beta}+\ln \theta\right)-\sum_{i=1}^{\mathrm{n}} \ln x_{i}-\sum_{i=1}^{\mathrm{n}} \frac{\ln \left(\theta x_{i}\right)}{\left(\theta x_{i}\right)^{-\beta}}+\sum_{i=1}^{\mathrm{n}} \frac{(p+\lambda) \ln \frac{\beta}{\theta}}{l_{i} s_{i}^{-1}\left(\frac{\theta}{x_{i}}\right)^{-\beta}} \\
& -(\mathrm{a}-1) \sum_{i=1}^{\mathrm{n}} \frac{\ln \frac{\beta}{\theta}\left[p s_{i}^{2}-k_{i}\right]}{k_{i}\left(\frac{\theta}{x_{i}}\right)^{-\beta}}+(2 \mathrm{a}-1) \sum_{i=1}^{\mathrm{n}} \frac{(1+\alpha) \ln \left(\frac{\theta}{x_{i}}\right)}{z_{i} s_{i}^{-1}\left(\frac{\theta}{x_{i}}\right)^{-\beta}} \\
& +\alpha(\mathrm{b}-1) \sum_{i=1}^{\mathrm{n}} \frac{l_{i}\left(\frac{\theta}{x_{i}}\right)^{\beta}\left(1-q_{i}\right)^{\frac{a-1}{a} \ln \left(\frac{\theta}{x_{i}}\right)}}{q_{i} s_{i}^{-1} z_{i}^{3}}, \\
& \frac{\partial \ell}{\partial \theta}=\frac{n \beta}{\theta}-\beta \theta^{\beta-1} \sum_{i=1}^{\mathrm{n}} x_{i}^{\beta}+\sum_{i=1}^{\mathrm{n}} \frac{(p+\lambda) \frac{\beta}{\theta}}{l_{i} s_{i}^{-1}\left(\frac{\theta}{x_{i}}\right)^{-\beta}}+(a-1) \frac{\beta}{\theta} \sum_{i=1}^{\mathrm{n}} \frac{\left(p s_{i}^{2}-k_{i}\right)}{k_{i}\left(\frac{\theta}{x_{i}}\right)^{-\beta}} \\
& +(1-\alpha)(2 a-1) \sum_{i=1}^{\mathrm{n}} \frac{\frac{\beta}{\theta}\left(\frac{\theta}{x_{i}}\right)^{\beta}}{z_{i} s_{i}^{-1}}+a \alpha \beta(b-1) \sum_{i=1}^{\mathrm{n}} \frac{l_{i}\left(1-q_{i}\right)^{\frac{a-1}{a}}}{\theta q_{i} s_{i}^{-1} z_{i}^{3}\left(\frac{\theta}{x_{i}}\right)^{-\beta}}, \\
& \frac{\partial \ell}{\partial a}=\frac{n}{a}+\sum_{i=1}^{\mathrm{n}} \ln k_{i}-2 \sum_{i=1}^{\mathrm{n}} \ln z_{i}+(\mathrm{b}-1) \sum_{i=1}^{\mathrm{n}} \frac{\left(2 \ln z_{i}-\ln k_{i}\right)}{q_{i}\left(1-q_{i}\right)^{-1}}
\end{aligned}
$$


$\frac{\partial \ell}{\partial \lambda}=\sum_{i=1}^{\mathrm{n}} \frac{\alpha-(\alpha+1) s_{i}}{l_{i}}+(\mathrm{a}-1) \sum_{i=1}^{\mathrm{n}} \frac{\alpha\left(1-s_{i}\right)}{k_{i} s_{i}^{-1}}+a \alpha(\mathrm{b}-1) \sum_{i=1}^{\mathrm{n}} \frac{\left(1-q_{i}\right)^{\frac{a-1}{a}}}{q_{i} s_{i}^{-1} z_{i}^{2}\left(s_{i}-1\right)^{-1}}$

and

$$
\frac{\partial \ell}{\partial b}=\frac{n}{b}+\sum_{i=1}^{\mathrm{n}} \ln q_{i}
$$

where $d_{i}=\alpha(\lambda+1) s_{i}-2 k_{i}$.

We can find the estimates of the unknown parameters by setting the score vector to zero, $\mathbf{U}(\widehat{\phi})=\mathbf{0}$, and solving them simultaneously yields the ML estimators $\hat{\alpha}, \hat{\beta}, \hat{\theta}, \hat{\lambda}$, $\hat{a}$ and $\hat{b}$. These equations cannot be solved analytically and statistical software can be used to solve them numerically by means of iterative techniques such as the Newton-Raphson algorithm. For the five parameters Kw-TMOF distribution all the second order derivatives exist. Setting these above equations to zero and solving them simultaneously also yield the MLEs of the six parameters.

For interval estimation of the model parameters, we require the $6 \times 6$ observed information matrix $J(\phi)=\left\{J_{r s}\right\}$ (for $\left.r, s=\alpha, \beta, \theta, \lambda, a, b\right)$ given in Appendix A. Under standard regularity conditions, the multivariate normal $N_{6}\left(0, J(\widehat{\phi})^{-1}\right)$ distribution can be used to construct approximate confidence intervals for the model parameters. Here, $J(\widehat{\phi})$ is the total observed information matrix evaluated at $\widehat{\phi}$. Therefore, Approximate $100(1-$ $\phi) \%$ confidence intervals for $\alpha, \beta, \theta, \lambda, a$ and $b$ can be determined as:

$\hat{\alpha} \pm Z_{\frac{\Phi}{2}} \sqrt{\hat{J}_{\alpha \alpha}}, \quad \hat{\beta} \pm Z_{\frac{\Phi}{2}} \sqrt{\hat{J}_{\beta \beta}}, \hat{\theta} \pm Z_{\frac{\Phi}{2}} \sqrt{\hat{J}_{\theta \theta}}, \quad \hat{\lambda} \pm Z_{\frac{\Phi}{2}} \sqrt{\hat{J}_{\lambda \lambda}}, \quad \hat{a} \pm Z_{\frac{\Phi}{2}} \sqrt{\hat{J}_{a a}}$ and $\hat{b} \pm Z_{\frac{\Phi}{2}} \sqrt{\hat{J}_{b b}}$,

where $Z_{\frac{\phi}{2}}$ is the upper $\phi$ th percentile of the standard normal distribution.

\section{Data Analysis}

In this section, we provide two applications of the $\mathrm{Kw}$-TMOF distribution to show its importance. We now provide a data analysis in order to assess the goodness-of-fit of the new model. For the two real data sets we shall compare the fits of the Kw-TMOF model with six of its sub models: the KMOIE, KMOIR, TMOF, MOF, TF and Fréchet distributions to show the potential of the new distribution. Moreover, we shall compare the proposed distribution with two non-nested models: gamma extended Fréchet (GEF) (da Silva et al., 2013) and beta Fréchet (BF) (Barreto-Souza et al., 2011) distributions with corresponding densities given (for $x>0$ ) by

$$
\begin{aligned}
& \text { BFr: } f(x)=\frac{\beta \theta^{\beta}}{B(a, b)} x^{-\beta-1} e^{-a\left(\frac{\theta}{x}\right)^{\beta}}\left[1-e^{-\left(\frac{\theta}{x}\right)^{\beta}}\right]^{b-1} \\
& \text { GEFr: } f(x)=\frac{a \beta \theta^{\beta}}{\Gamma(b)} x^{-\beta-1} e^{-\left(\frac{\theta}{x}\right)^{\beta}}\left[1-e^{-\left(\frac{\sigma}{x}\right)^{\beta}}\right]^{a-1}\left\{-\log \left[1-e^{-\left(\frac{\theta}{x}\right)^{\beta}}\right]^{a}\right\}^{b-1},
\end{aligned}
$$

where $\beta, \theta, a$ and $b$ are positive parameters. 


\section{Data Set I: Glass Fibres}

The first data set is obtained from Smith and Naylor (1987). The data are the strengths of $1.5 \mathrm{~cm}$ glass fibres, measured at the National Physical Laboratory, England. Unfortunately, the units of measurement are not given in the paper.

\section{Data Set II: Carbon Fibres}

The second data set corresponds to an uncensored data set from Nichols and Padgett (2006) on breaking stress of carbon fibres (in Gba). This data set was previously studied by Afify et al. (2014) to fit the transmuted complementary Weibull geometric distribution.

Table 2: The statistics $-2 \widehat{\ell}, A I C, B I C, H Q I C$ and $C A I C$ for data set I

\begin{tabular}{|c|c|c|c|c|c|}
\hline Model & $-2 \hat{\ell}$ & $A I C$ & $B I C$ & $H Q I C$ & $C A I C$ \\
\hline Kw-TMOF & 43.775 & 55.775 & 68.634 & 60.833 & 57.275 \\
\hline TMOF & 48.46 & 56.46 & 65.032 & 59.831 & 57.149 \\
\hline KTMOIE & 54.572 & 64.572 & 75.287 & 68.786 & 65.624 \\
\hline BF & 60.63 & 68.63 & 77.202 & 72.002 & 69.32 \\
\hline GEF & 61.557 & 69.557 & 78.13 & 72.929 & 70.247 \\
\hline MOF & 95.738 & 101.738 & 108.168 & 104.267 & 102.145 \\
\hline TF & 94.078 & 100.078 & 106.507 & 102.606 & 100.484 \\
\hline F & 93.707 & 97.707 & 101.993 & 99.392 & 97.907 \\
\hline
\end{tabular}

Table 3: MLEs and their standard errors (in parentheses) for data set I

\begin{tabular}{|c|c|c|c|c|c|c|}
\hline Model & \multicolumn{7}{|c|}{ Estimates } \\
\hline & $\hat{\alpha}$ & $\hat{\beta}$ & $\hat{\theta}$ & $\hat{\lambda}$ & $\hat{a}$ & $\hat{b}$ \\
\hline Kw-TMOF & 1.0017 & 0.9451 & 2.3330 & $-5.4912 \times 10^{-5}$ & 3.4721 & 142.4414 \\
\hline & $(0.961)$ & $(0.244)$ & $(1.498)$ & $(0.024)$ & $(0.046)$ & $(9.162)$ \\
\hline TMOF & 376.268 & 6.8744 & 0.65 & 0.1499 & -- & -- \\
\hline & $(246.832)$ & $(0.596)$ & $(0.049)$ & $(0.302)$ & -- & -- \\
\hline KTMOIE & 0.4057 & -- & 1.8859 & $-10 \times 10^{-5}$ & 4.788 & 21.8011 \\
\hline & $(0.064)$ & -- & $(0.766)$ & $(0.396)$ & $(2.401)$ & $(8.284)$ \\
\hline BF & -- & 0.6466 & 2.0518 & -- & 15.0756 & 36.9397 \\
\hline & -- & $(0.163)$ & $(0.986)$ & -- & $(12.057)$ & $(22.649)$ \\
\hline GEF & -- & 0.7421 & 1.6625 & -- & 32.112 & 13.2688 \\
\hline & -- & $(0.197)$ & $(0.952)$ & -- & $(17.397)$ & $(9.967)$ \\
\hline MOF & 0.4816 & 2.3876 & 1.5441 & -- & -- & -- \\
\hline & $(0.252)$ & $(0.253)$ & $(0.226)$ & -- & -- & -- \\
\hline TF & -- & 2.7898 & 1.3068 & 0.1298 & -- & -- \\
\hline & -- & $(0.165)$ & $(0.034)$ & $(0.208)$ & -- & -- \\
\hline F & -- & 2.8876 & 1.2643 & -- & -- & -- \\
\hline & -- & $(0.234)$ & $(0.059)$ & -- & -- & -- \\
\hline
\end{tabular}


Table 4: The statistics $-2 \widehat{\ell}, A I C, B I C, H Q I C$ and $C A I C$ for data set II

\begin{tabular}{|c|c|c|c|c|c|}
\hline Model & $-2 \hat{\ell}$ & $A I C$ & $B I C$ & $H Q I C$ & $C A I C$ \\
\hline Kw-TMOF & 291.292 & 303.292 & 318.923 & 309.618 & 304.195 \\
\hline KTMOIE & 301.016 & 311.016 & 324.022 & 316.288 & 311.645 \\
\hline BF & 303.133 & 311.133 & 321.553 & 315.35 & 311.554 \\
\hline GEF & 303.96 & 311.96 & 332.381 & 316.178 & 312.381 \\
\hline TMOF & 301.973 & 309.973 & 320.393 & 314.19 & 310.394 \\
\hline MOF & 345.328 & 351.328 & 359.143 & 354.491 & 351.578 \\
\hline TF & 344.475 & 350.475 & 358.29 & 353.638 & 350.725 \\
\hline F & 344.308 & 348.308 & 353.519 & 350.417 & 348.432 \\
\hline
\end{tabular}

\section{Model Selection}

The model selection is carried out using goodness-of-fit measures including the Akaike information criterion $(A I C)$, consistent Akaike information criterion $(C A I C)$, Bayesian information criterion $(B I C)$, Hannan-Quinn information criterion $(H Q I C)$ and $-2 \hat{\ell}$ (where $\hat{\ell}$ is the maximized log-likelihood).

Tables 2 and 4 list the numerical values of the $-2 \hat{\ell}, A I C, B I C, H Q I C$ and $C A I C$ using the first data set, whilst the MLEs and their corresponding standard errors (in parentheses) of the model parameters are shown in tables 3 and 5. These numerical results are obtained using the Mathcad program. Based on these criteria in tables 2 and 4, we conclude that the Kw-TMOF distribution provides a superior fit to these data than its sub models and non-nested models. Figures 3 and 4 display the fitted pdf and cdf the Kw-TMOF model to both data sets. It is clear from these plots that the $\mathrm{Kw}$-TMOF provides close fit to the two data sets.

Table 5: MLEs and their standard errors (in parentheses) for data set II

\begin{tabular}{|c|c|c|c|c|c|c|}
\hline Model & \multicolumn{7}{|c|}{ Estimates } \\
\hline & $\hat{\alpha}$ & $\hat{\beta}$ & $\hat{\theta}$ & $\hat{\lambda}$ & $\hat{a}$ & $\hat{b}$ \\
\hline Kw-TMOF & 1.0766 & 0.5893 & 4.5252 & $-5.5611 \times 10^{-5}$ & 3.0559 & 67.2623 \\
\hline & $(0.53)$ & $(0.088)$ & $(5.293)$ & $(0.509)$ & $(1.669)$ & $(46.922)$ \\
\hline KTMOIE & 1.4565 & -- & 2.9071 & -0.0002 & 1.7674 & 9.6266 \\
\hline & $(0.416)$ & -- & $(1.967)$ & $(0.336)$ & $(0.892)$ & $(2.585)$ \\
\hline BF & -- & 0.4046 & 1.6097 & -- & 22.0143 & 29.7617 \\
\hline & -- & $(0.108)$ & $(2.498)$ & -- & $(21.432)$ & $(17.479)$ \\
\hline GEF & -- & 0.4776 & 1.3692 & -- & 27.6452 & 17.4581 \\
\hline & -- & $(0.133)$ & $(2.017)$ & -- & $(14.136)$ & $(14.818)$ \\
\hline TMOF & 101.923 & 3.3313 & 0.6496 & 0.2936 & -- & -- \\
\hline & $(47.625)$ & $(0.206)$ & $(0.068)$ & $(0.27)$ & -- & -- \\
\hline MOF & 0.5988 & 1.5796 & 2.3066 & -- & -- & -- \\
\hline & $(0.3091)$ & $(0.16)$ & $(0.498)$ & -- & -- & -- \\
\hline TF & -- & 1.7435 & 1.9315 & 0.0819 & -- & -- \\
\hline & -- & $(0.076)$ & $(0.097)$ & $(0.198)$ & -- & -- \\
\hline F & -- & 1.7766 & 1.8705 & -- & -- & -- \\
\hline & -- & $(0.113)$ & $(0.112)$ & -- & -- & -- \\
\hline
\end{tabular}




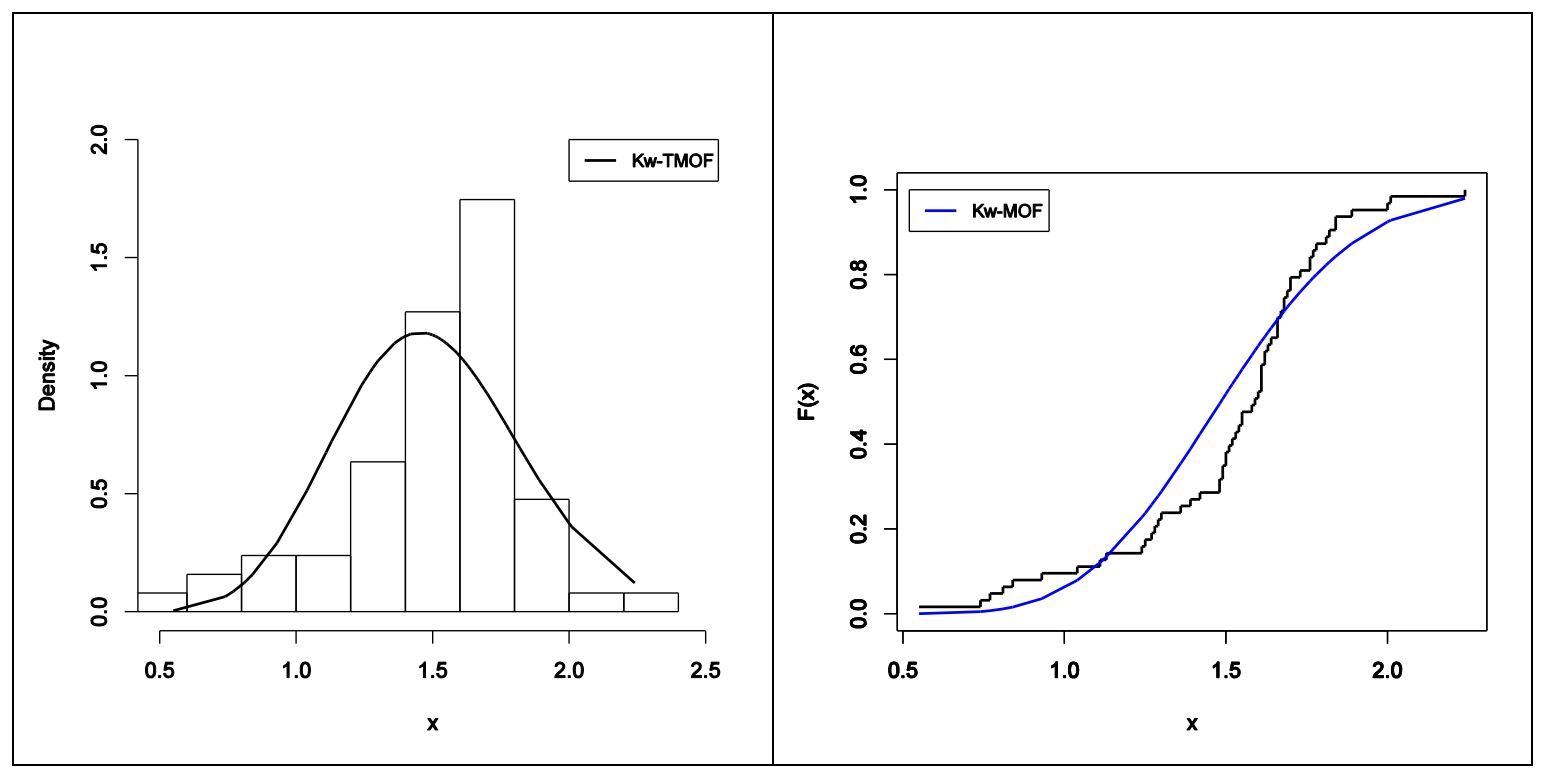

Figure 3: The estimated pdf and cdf of the Kw-TMOF model for data set I.

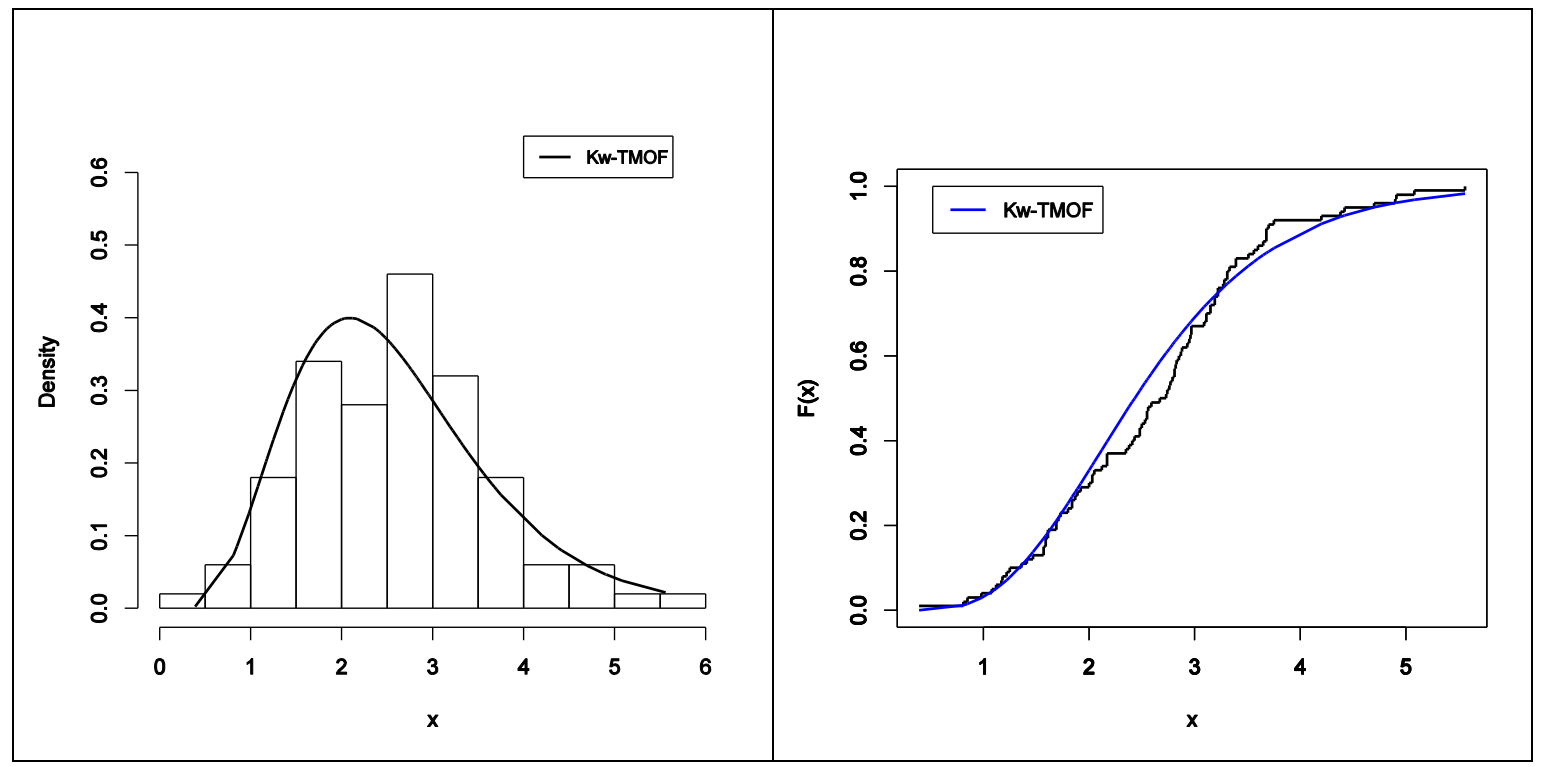

Figure 4: The estimated pdf and cdf of the Kw-TMOF model for data set II.

\section{Conclusions}

In this paper, We propose a new six-parameter distribution, called the Kumaraswamy transmuted Marshall-Olkin Fréchet (Kw-TMOF) distribution, which extends the transmuted Marshall-Olkin Fréchet (TMOF) distribution (Afify et al., 2015). We provide some of its mathematical and statistical properties. The Kw-TMOF density function can be expressed as a mixture of Fréchet densities. We derive explicit expressions for the ordinary and incomplete moments, Rényi and $\eta$-entropies. We also obtain the density function of the order statistics and their moments. We discuss maximum likelihood 
estimation and calculate the information matrix. Two applications illustrate that the KwTMOF distribution provides better fit than other competitive distributions. We hope that the proposed extended model may attract wider applications in survival analysis.

\section{References}

1. Afify, A. Z., Hamedani, G. G., Ghosh, I. and Mead, M. E. (2015). The transmuted Marshall-Olkin Fréchet distribution: properties and applications. International Journal of Statistics and Probability, 4, 132-184.

2. Afify, A. Z., Yousof, H. M., Cordeiro, G. M., Ortega, E. M. M. and Nofal, Z. M. (2016). The Weibull Fréchet distribution and its applications. Journal of Applied Statistics, forthcoming.

3. Afify, A. Z., Yousof, H. M., Ahmad, M. and Ahmed, A. N. (2015). The Kumaraswamy Marshall-Olkin Fréchet distribution with applications. Submitted.

4. Afify, A. Z., Nofal, Z. M. and Butt, N. S. (2014). Transmuted complementary Weibull geometric distribution. Pak. J. Stat. Oper. Res., 10, 435-454.

5. Ahmad, A., Ahmad, S. P. and Ahmed, A. (2014). Transmuted inverse Rayleigh distribution: a generalization of the inverse Rayleigh distribution. Mathematical Theory and Modeling, 4, 90-98.

6. Barlow, R. E. and Proschan, F. (1981). Mathematical theory of reliability, 17. Siam.

7. Barreto-Souza, W. M., Cordeiro, G. M. and Simas, A. B. (2011). Some results for beta Fréchet distribution. Commun. Statist. Theory-Meth., 40, 798-811.

8. Bjerkedal, T. (1960). Acquisition of resistance in guinea pigs infected with different doses of virulent tubercle bacilli.Amer. J. Hyg.72:130-148.

9. Cordeiro, G. M. and de Castro, M. (2011). A new family of generalized distributions. Journal of Statistical Computation and Simulation, 81, 883-898.

10. da Silva, R. V., de Andrade, T. A., Maciel, D., Campos, R. P. and Cordeiro, G. M. (2013). A new lifetime model: the gamma extended Fréchet distribution. Journal of Statistical Theory and Applications, 12, 39-54.

11. de Gusmão, F. R., Ortega, E. M. and Cordeiro, G. M. (2011). The generalized inverse Weibull distribution. Statistical Papers, 52, 591-619.

12. Elbatal, I. Asha, G. and Raja, V. (2014). transmuted exponentiated Fréchet distribution: properties and applications. J. Stat. Appl. Pro., 3, 379-394.

13. Fréchet, M. (1924). Sur la loi des erreurs dobservation. Bulletin de la Société Mathématique de Moscou, 33, 5-8.

14. Glänzel, W. (1987). A characterization theorem based on truncated moments and its application to some distribution families. Mathematical Statistics and Probability Theory (Bad Tatzmannsdorf, 1986), Vol. B, Reidel, Dordrecht, 75-84.

15. Glänzel, W. (1990). Some consequences of a characterization theorem based on truncated moments. Statistics: A Journal of Theoretical and Applied Statistics, 21, 613-618. 
16. Keller, A. Z. and Kamath, A. R. (1982). Reliability analysis of CNC Machine Tools. Reliability Engineering, 3, 449-473.

17. Krishna, E., Jose, K. K., Alice, T. and Ristic', M. M. (2013). The Marshall-Olkin Fréchet distribution. Communications in Statistics-Theory and Methods, 42, 4091-4107.

18. Kotz, S. and Nadarajah, S. (2000). Extreme value distributions: theory and applications. Imperial College Press, London.

19. Mahmoud, M.R. and Mandouh, R.M. (2013). On the transmuted Fréchet distribution. Journal of Applied Sciences Research, 9, 5553-5561.

20. Mead, M.E. and Abd-Eltawab A.R. (2014). A note on Kumaraswamy-Fréchet distribution. Aust. J. Basic and Appl. Sci., 8, 294-300.

21. Mead, M. E., Afify, A. Z., Hamedani, G. G. and Ghosh, I. (2016). The beta exponential Fréchet distribution with applications. Austrian Journal of Statistics, forthcoming.

22. Merovci, F., Elbatal, I. and Ahmed, A. (2013). Transmuted generalized inverse Weibull distribution. arXiv preprint arXiv:1309.3268.

23. Nadarajah, S. and Gupta, A.K. (2004). The beta Fréchet distribution. Far East Journal of Theoretical Statistics, 14, 15-24.

24. Nadarajah, S. and Kotz, S. (2003). The exponentiated exponential distribution. Available online at http://interstat.stat journals.net/YEAR/2003/abstracts/ 0312001.php.

25. Nichols, M. D. and Padgett, W. J. (2006). A bootstrap control chart for Weibull percentiles. Quality and Reliability Engineering International, 22, 141-151.

26. Oguntunde, P. E. and Adejumo, A. O. (2015). The transmuted inverse exponential distribution. International Journal of Advanced Statistics and Probability, 3, 1-7.

27. Shahbaz, M. Q., Shahbaz, S. and Butt, N. S. (2012). The Kumaraswamy--inverse Weibull distribution. Pak. J. Stat. Oper. Res., 8, 479-489.

28. Smith, R. L. and Naylor, J. C. (1987). A comparison of maximum likelihood and Bayesian estimators for the three-parameter Weibull distribution. Appl. Statist., 36, 358-369.

29. Treyer, V. N. (1964). Doklady Acad, Nauk, Belorus, U.S.S.R.

30. Yousof, H. M., Afify, A. Z., Alizadeh, M., Butt, N. S., Hamedani, G. G. and Ali, M. M. (2015). The transmuted exponentiated generalized-G family of distributions. Pak. J. Stat. Oper. Res., 11, 441-464. 